\title{
A PROPÓSITO DE UN CASO: "LA NIÑA QUE SUDA SANGRE: UN RARO CASO DE HEMATOHIDROSIS"
}

\author{
About a case: \\ The girl who sweats blood: A rare case of hematohidrosis
}

\section{Zuleika Morillo ${ }^{1 *}$, Wirson Ureña Correa ${ }^{1 * *}$, Deseado S. Guzmán ${ }^{1 * * *}$, Yosira Ortiz ${ }^{1 * * * *}$}

Recibido: enero 23, 2019 • Aprobado: marzo 6, 2019

Cómo citar: Morillo Z, Ureña Correa W, Guzmán DS, Ortiz Y. A propósito de un caso: "la niña que suda sangre: un raro caso de hematohidrosis". cysa [Internet]. 26 de julio de 2019 [citado 26 de julio de 2019];3(2): 65-69. Disponible en:

https://revistas.intec.edu.do/index.php/cisa/article/view/1480

\section{Resumen}

Introducción: la hematohidrosis es una rara condición clínica donde el paciente exuda sangre.

Presentación de caso: se presenta el caso de una joven de 13 ańos, sin antecedentes mórbidos conocidos, que fue referida por Departamento de Hematología a Salud Mental por presentar cuadro de 7 meses de evolución, caracterizado por sangrado de oídos, ojos, boca, ombligo, cabeza, mamas y dedos, ocurriendo con más frecuencia cuando cursa con períodos menstruales.

Se le realizaron analíticas y estudios especializados que incluían hemograma, glicemia, perfil renal, hepático, virales, electrolitos, perfil tiroideo, uroanálisis, Factor viII, Factor VII, Factor de Von Willebrand, TP, TTP, INR, fibrinógeno y extendido de sangre periférica. Además, se realizó TAC de cráneo y radiografía de senos paranasales. Todas las pruebas estaban dentro de los parámetros de normalidad. Se inicia tratamiento con Hidroxicina $25 \mathrm{mg}$ c/24 horas y clonazepam $2 \mathrm{mg}$ c $/ 24$ horas. Con el tratamiento farmacológico cedió el sangrado de la paciente.

Conclusión: el paciente fue diagnosticado con hematohidrosis, clínicamente, por exclusión. Actualmente no hay tratamiento disponible para esta condición. La etiología es desconocida hasta la fecha. El estrés se vincula como un factor precipitante.

Palabras Clave: hematohidrosis; sangrado; estrés; fibrinógeno; Von Willebrand.

1. Departamento de Salud Mental, Hospital Infantil Dr. Robert Reid Cabral

\begin{abstract}
Introduction: Hematohidrosis is a rare clinical condition where the patient exudes blood.

Presentation: We present the case of a 13-year-old girl, with no known morbid history, who was referred by department of hematology to mental health for presenting a 7-month evolution, characterized by bleeding of ears, eyes, mouth, navel, head, breasts and fingers occurring more often when going through menstrual periods.

Analyzes and specialized studies were performed that included hemogram, glycemia, renal, hepatic, viral, electrolyte, thyroid profile, uroanalysis, Factor VIII, Factor VII, Von Willebrand factor, TP, TTP, INR, fibrinogen and peripheral blood smear. In addition, a CT scan of the skull and $\mathrm{x}$-ray of the paranasal sinuses was performed. All the tests were within normal parameters. Treatment was started with Hydroxyzine $25 \mathrm{mg}$ every 24 hours and clonazepam $2 \mathrm{mg}$ every 24 hours. With the pharmacological treatment, the bleeding of the patient subsided.
\end{abstract}

Conclusion: The patient was diagnosed with hematohidrosis clinically by exclusion. Currently, there is no treatment available for this condition. The etiology is unknown to date. Stress is linked as a precipitating factor.

Keywords: Hematohidrosis; bleeding; stress; fibrinogen; Von Willebrand.

\footnotetext{
*paidoszu@hotmail.com

** dr.wurenacorrea@gmail.com

***deseado@gmail.com

****draortizdelacruz@gmail.com
} 


\section{Introducción}

La hematohidrosis es una rara condición clínica donde el individuo exuda sangre. Puede ocurrir cuando una persona sufre de estrés extremo. Se han informado muy pocos casos de hematohidrosis en la literatura ${ }^{1}$. La hematohidrosis también se conoce como hematidrosis y hemidrosis. La etiología exacta de la hematohidrosis es desconocida. Se cree que es una enfermedad sistémica. Se ha informado hematohidrosis con púrpura trombocitopénica primaria. En otro estudio, se informó un caso de hematohidrosis, acompañado con otorrea y otoeritrosis ${ }^{2}$.

La hematohidrosis también puede ocurrir en entorno de estrés extremo, cuando el paciente esté enfrentando situaciones que detonen ansiedad o síntomas afectivos $^{3}$.

Pocas teorías se han propuesto con respecto a la etiopatogenia de la hematohidrosis. Una de esas escuelas de pensamiento dice que hay múltiples vasos sanguíneos alrededor de las glándulas sudoríparas dispuestos en forma de red. Se cree que bajo la presión de un gran estrés los vasos se contraen ${ }^{4}$. Posteriormente, a medida que pasa la ansiedad, los vasos sanguíneos se dilatan hasta su ruptura. La sangre en este punto entra en las glándulas sudoríparas que empujan la misma sangre hacia la superficie y se manifiesta como gotas de sangre mezcladas con sudor ${ }^{5}$. El término "hematofolliculohidrosis" se propuso porque aparecía junto con un líquido similar al sudor y la sangre se exudaba a través de los canales foliculares.

Se realizó una búsqueda de los casos reportados en la red, tanto por los médicos como por los mismos pacientes, a nivel mundial, con un total de 56 casos; fueron estudiados y clasificados en categorías de acuerdo con el factor causal. Esto es: componente de la enfermedad sistémica, menstruación indirecta, esfuerzo excesivo, psicógeno y desconocido. Los psicógenos se subdividieron en aquellos que ocurrieron solo una vez, los que recurrieron en repetidas ocasiones y los estigmatizados. El miedo agudo y la ansiedad intensa fueron las causas incitadoras más frecuentes ${ }^{6}$. La hematohidrosis es un fenómeno clínico extremadamente raro.

\section{Caso clínico}

Reportamos el caso de una adolescente de 13 años, quien es referida al Departamento de Salud Mental, Hospital Infantil Dr. Robert Reid Cabral, por hematología, con historia de siete meses de emisión de sangre a través de oídos, boca, ojos, ombligo, mamas y dedos; ocurriendo con más frecuencia cuando cursaba con períodos menstruales, dicho cuadro se había incrementado en los últimos meses. Al evaluar la paciente nos encontramos como hallazgos relevantes en examen físico: secreciones en forma de sangre roja, dolor en oído no especificado, lágrimas rojas, dolor abdominal leve a la palpación, evacuaciones diarreicas sanguinolentas, lesión tipo hematoma en ambos brazos y sudoración profusa.

Foto 1. Exudado por el ombligo

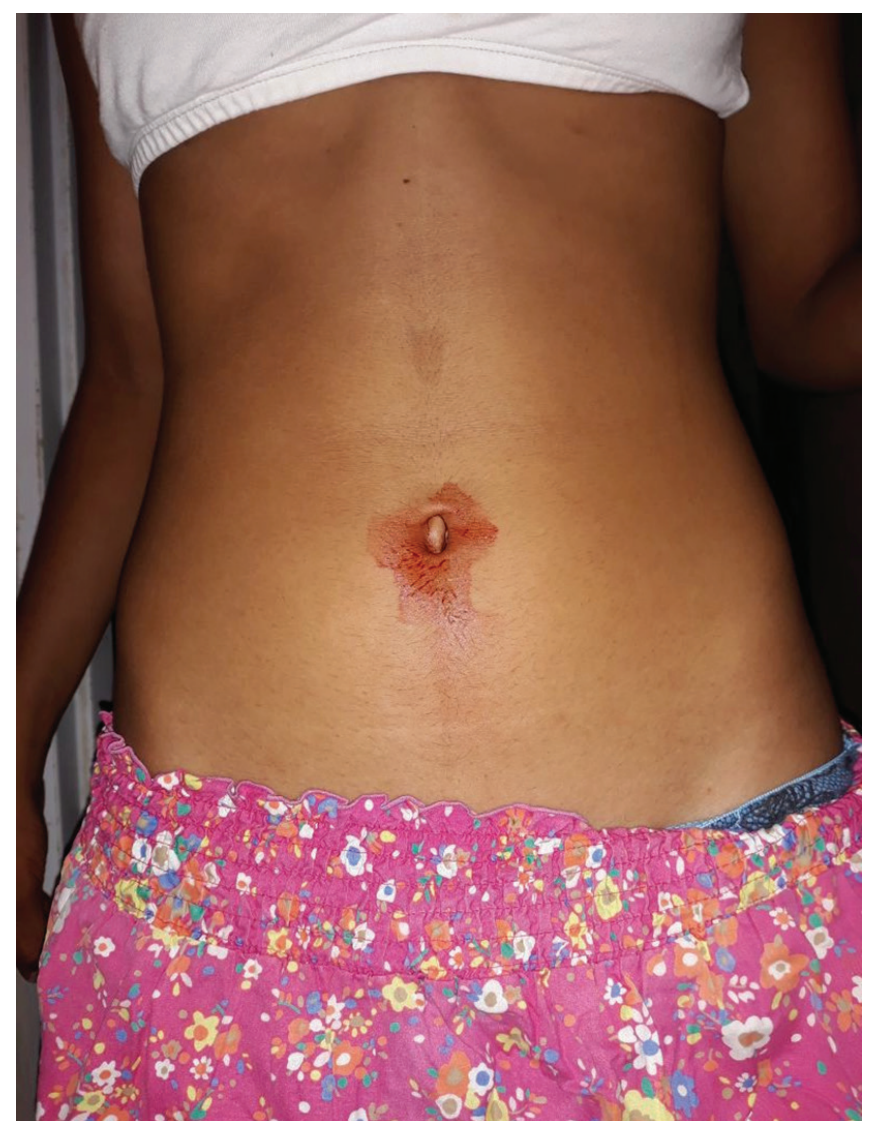


Foto 2. Exudado por el oído

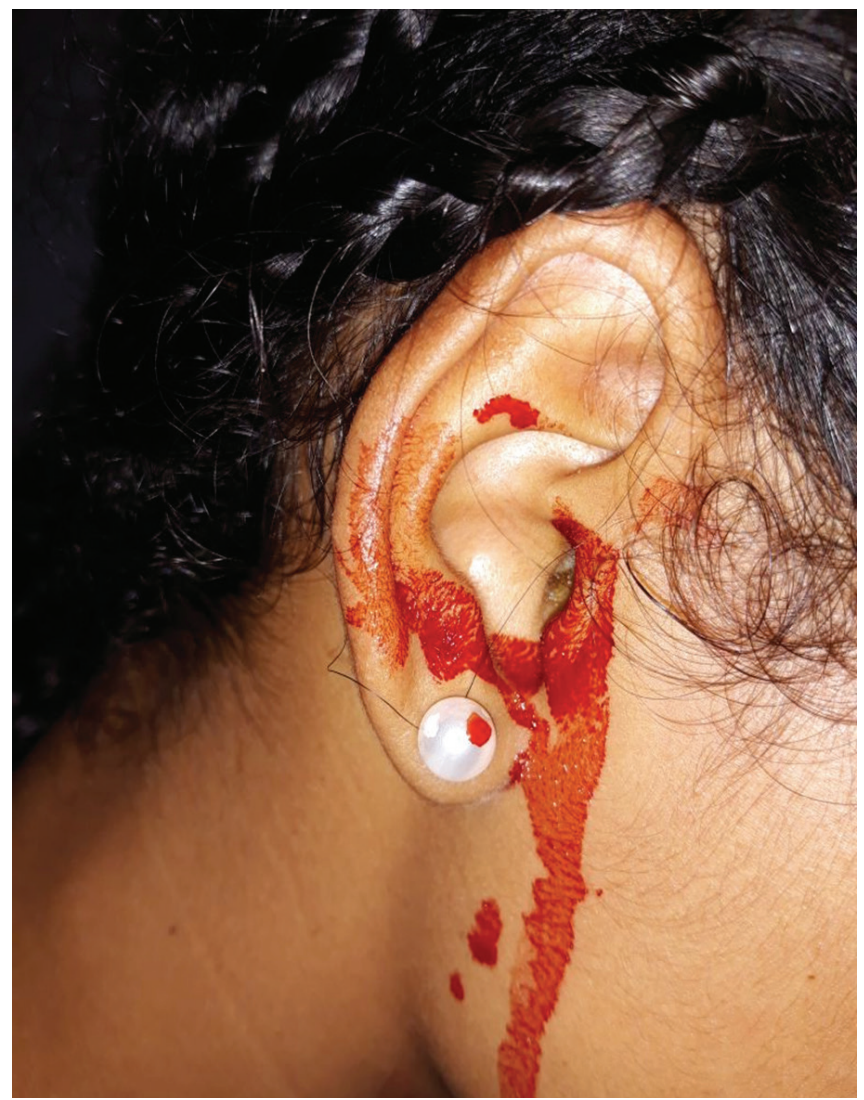

Fuente: fotos de la paciente al momento de la entrevista.

En el examen mental nos encontramos con una paciente con una conducta adecuada, que recibía tratamiento con Amchafibrina $500 \mathrm{mg}$ c/24 horas por hematología. Como antecedentes personales patológicos la paciente presentaba asma bronquial desde los 6 años con exacerbaciones frecuentes y múltiples procesos gripales a repetición, trombocitopenia no especificada a la edad de 10 años, Salmonelosis a la edad de 10 años y amebiasis intestinal a la edad de 10 ańos. $\mathrm{Al}$ interrogar a la madre sobre la historia del embarazo y concepción nos refiere que fue un embarazo no deseado, intentó abortarla, contó con chequeos irregulares solo en el tercer trimestre, presentó hipertensión gestacional y consumió alcohol y tabaco durante el embarazo. A pesar de esto la joven evidenció un desarrollo aparente normal, en la actualidad vivía con su abuela. Como antecedentes psiquiátricos familiares refieren que la abuela materna presenta cuadros de inestabilidad emocional, sin precisar síntomas psiquiátricos.

Se le realizan a la paciente analíticas y estudios especializados que incluían hemograma, glicemia, perfil renal, hepático, virales, electrolitos, perfil tiroideo, uroanalisis, Factor viII, Factor viI, Factor de Von Willebrand, TP, TTP, INR, fibrinógeno y extendido de sangre periférica. Además, se realizó TAC de cráneo y radiografía de senos paranasales. Todos dentro de parámetros de normalidad. Nos planteamos el diagnóstico de Hematohidrosis probable y descartar trastorno de ansiedad. Decidimos iniciar medicación con hidroxicina $25 \mathrm{mg}$ c/ 24 horas y clonazepam $2 \mathrm{mg}$ c/24 horas, con lo que cedió el sangrado en la paciente.

La paciente seguía consultas irregulares con Departamento de Psiquiatría. Presentando una exacerbación 2 años más tarde, posterior a la muerte de su abuela, mudanza, cambio de escuela y ruptura con pareja actual. En esta exacerbación además la joven presentaba nueve meses sin adherencia medicamentosa. Este cuadro fue tratado con benzodiacepinas tipo clonazepam $2 \mathrm{mgc} / 24$ horas presentando la misma remisión del cuadro de sangrado.

\section{Discusión}

Nos encontramos ante el primer caso de hematohidrosis registrado en nuestro país y ante una patología muy poco frecuente, con pocos casos a nivel mundial.

La hematohidrosis, también conocida como hematidrosis, hemidrosis y hematidrosis, es una afección en la cual los vasos sanguíneos capilares que alimentan las glándulas sudoríparas se rompen, provocando que exuden sangre, y ocurre en condiciones de estrés físico o emocional extremo ${ }^{7}$.

Aunque no existe una causa definida de esta rara patología los casos existentes vinculan la gran relación con síntomas psiquiátricos, sobre todo con 
situaciones que detonen gran ansiedad. En un informe de un caso en la India, la causa probable de la hematohidrosis fue el estrés crónico, ya que las otras causas fueron descartadas mediante investigaciones detalladas. También se cree que los trastornos de ansiedad y trastornos afectivos inducen el sangrado. Se supone que la púrpura psicógena es causada por la hipersensibilidad a la propia sangre de los pacientes o a la sensibilización de los autoeritrocitos y se caracteriza por cultivos repetidos de equimosis, hemorragias gastrointestinales y hematuria ${ }^{8}$.

La sangre extravasada tiene componentes celulares idénticos a los de la sangre periférica. La ansiedad mental severa activa el sistema nervioso simpático para invocar la reacción de estrés o lucha, a tal punto que causa una hemorragia de los vasos que irrigan las glándulas sudoríparas ${ }^{9}$. El efecto sobre el cuerpo es la debilidad y la deshidratación leve a moderada, debido a la ansiedad severa y a la pérdida de sangre y sudor ${ }^{10}$.

Es necesario hacer un análisis de la historia de la paciente para entender con claridad cómo se ha podido detonar esta patología en ella.

Es conveniente recordar cómo el proceso de desarrollo biopsicosocial de un ser humano afecta sus interacciones futuras, estabilidad emocional y puede, si no ocurre en circunstancias adecuadas, ser el gestor de patologías psiquiátricas.

Por desarrollo biopsicosocial entendemos los cambios progresivos, sujetos a leyes y principios. Comienza en la concepción y culmina en la madurez. Su objetivo es la adquisición de una entidad biológica, psicológica y social que equilibre las necesidades del individuo con el contexto social en el que esté inserto ${ }^{11}$.

Desde la perspectiva psicodinámica el desarrollo de un individuo comienza con la historia de sus padres, sigue con el deseo de tener un hijo y continúa con la construcción del niño imaginario a través de ideas proyectadas de ambos padres. Un punto clave es la relación materno-fetal que se establece a través de la conexión humoral y sensorial. En el caso antes expuesto tenemos una paciente que no contó con un proceso de desarrollo biopsicosocial adecuado, sin una relación materno-fetal saludable, no fue deseada por su madre y algo relevante es que su madre presentó un episodio depresivo en el embarazo, lo cual afecta el adecuado desarrollo fetal y predispone al bebé a psicopatología en edades ulteriores ${ }^{4,5}$.

Nos encontramos en este caso con una adolescente que no tuvo un apego seguro con su madre y que fue criada por su abuela. Es una paciente con un probable antecedente familiar de enfermedad psiquiátrica y que al momento de los cuadros de sangrado tenía fuertes estresores que al parecer detonaban en la paciente ansiedad.

\section{Conclusión}

Al descartar todas las patologías de origen hematológicos y ante dos cuadros que cedieron adecuadamente con ansiolíticos proponemos el diagnóstico de hematohidrosis, un caso singular.

\section{Recomendaciones}

Ante futuros casos de pacientes con sangrado por piel y mucosas tener presentes este diagnóstico poco frecuente, el cual se hace por descarte de otras patologías hematológicas y otorrinolaringológicas.

\section{Bibliografía}

1. Patel R M, Mahajan S. Hematohidrosis: A rareclinicalentity. IndianDermatology Online Journal [Internet]. 2010 [consultada 10 de noviembre 2018];1(1): 30-2. Available from: http://doi. org/10.4103/2229-5178.73256.

2. Jayaraman A R, Kannan P, Jayanthini V. An interesting case report of Hematohidrosis. IndianJournal of Psychological Medicine [Internet]. 2017 [consultada 10 de diciembre 2018];39(1): 83-5. Available from: http://doi. org/10.4103/0253-7176.198953. 
3. Zhang FK, Zheng YL, Liu JH, Chen HS, Liu SH, XuMQ, etal. Clinical and laboratorystudyofa case of hematidrosis. ZhonghuaXue Ye XueZaZhi [Internet]. 2004 [consultada 28 de septiembre 2018];25: 147-50. Available from: https://www. ncbi.nlm.nih.gov/pubmed/24568922.

4. Wang Z, Yu Z, Su J, Cao L, Zhao X, Bai X, et al. A case of hematidrosis successfully treated with propranolol. Am J ClinDermatol [Internet]. 2010 [consultada 28 de septembre 2018];11: 440-3. doi: $10.2165 / 11531690-000000000-00000$.

5. Bhagwat PV, Tophakhane RS, Rathod RM, Shashikumar BM, Naidu V. Hematohidrosis. Indian J DermatolVenereolLeprol [Internet]. 2009 [consultada 10 de diciembre 2018];75: 317-8. doi: 10.4103 / 0378-6323.51267.

6. Holoubek JE, Holoubek AB. Blood, sweat and fear. "A classification of hematidrosis" J Med [Internet]. 1996 [consultada 10 de diciembre 2018];27: 115-33. Available from: https://www. ncbi.nlm.nih.gov/pubmed/.

7. Champion RH. Disorders of sweatglands. In: Champion RH, Burton JL, Burns DA, Breathnach SM (6th ed.). Rook's Textbook of Dermatology. London: Blackwell Science; 1998. pp. 2001-2.

8. Norn MS. Microscopically and chemically detected haemolacria. ActaOphthalmol (Copenh) [Internet]. 1977 [consultada 29 de noviembre 2018];55: 132-40. Available from: https://www. ncbi.nlm.nih.gov/pubmed/?

9. Tshifularo M. Bloodotorrhea: Blood stained sweat ear discharges: Hematohidrosis: four case series (2001-2013) Am J Otolaryngol [Internet]. 2014 [consultada 10 de diciembre 2018];35: 271-3. doi: 10.1016 / j.amjoto.2013.09.006.
10. Jerajani HR, Jaju B, Phiske MM, Lade N. Hematohidrosis - A rare clinical phenomenon. Indian J Dermatol [Internet]. 2009 [consultada 10 de diciembre 2018]; 54:290-2. doi: 10.4103 / 0019-5154.55645.

11. Almonte C, Motts E. Psicopatología Infantil y de la Adolescencia. Santiago de Chile: Editorial Mediterráneo; 2012.

12. Rydz N, James PD. Aproximación al diagnóstico y manejo de los trastornos hemorrágicos comunes. Semin Thromb Hemost [Internet]. 2012 [consultada 15 de octubre 2018];38(7): 711-9. Available from: /www.ncbi.nlm.nih.gov/pubmed?filters.

13. Maamoun A, Hazzaa A, Riad E. Un fenómeno fascinante: estudio de caso y descripción general de la literatura. Semin Thromb Hemost [Internet]. 2018 [consultada 20 de noviembre 2018]; 44 (3): 293-5. doi: 10.1055 / s-0037-1608905.

14. Kluger N. Hematidrosis (sudoración sanguínea): una revisión de la literatura reciente (1996-2016). Acta Dermatoverol Alp Pannonina [Internet]. 2018 [consultada 20 de noviembre 2018]; 27(2): 85-90. Available from: www.ncbi.nlm.nih.gov/ pubmed/29945265.

15. Praveen Bk, Vicente J. Hematidrosis y hemolacria: reporte de un caso. Indian J Pediatría [Internet]. 2012 [consultada 20 de noviembre 2018]; 79(1): 109-11. doi: 10.1007 / s12098-011-0449-2. 